Theorie : Gestaltung 
Das Institut für Theorie (ith) betreibt Grundlagen- und angewandte Forschung und entwickelt entlang aktueller ästhetischer Fragen ein Theorieverständnis, das in engem Bezug zur Praxis der Gestaltung und Kunst und deren gesellschaftlicher Relevanz steht. Die Arbeit ist transdisziplinär und auf Wissenstransfer und Vernetzung ausgerichtet.

T:G\01 Bettina Heintz / Jörg Huber (Hgg.), Mit dem Auge denken:

Strategien der Sichtbarmachung in wissenschaftlichen und virtuellen Welten.

T:G\02 Ursula Biemann (ed.), Stuff it: The Video Essay in the Digital Age.

T:G\03 Marion von Osten (Hg.), Norm der Abweichung.

T:G\04 Juerg Albrecht / Jörg Huber / Kornelia Imesch / Karl Jost / Philipp Stoellger (Hgg.), Kultur Nicht Verstehen: Produktives Nichtverstehen und Verstehen als Gestaltung.

T:G\05 Jörg Huber / Philipp Stoellger / Gesa Ziemer / Simon Zumsteg (Hgg.), Ästhetik der Kritik, oder: Verdeckte Ermittlung.

T:G\06 Jörg Huber / Philipp Stoellger (Hgg.), Gestalten der Kontingenz: Ein Bilderbuch.

T:G\07 Jörg Huber / Gesa Ziemer / Simon Zumsteg (Hgg.), Archipele des Imaginären.

Die Publikationsreihe T:G (Theorie:Gestaltung) wird realisiert als Koproduktion des Instituts für Theorie (ith) und Edition Voldemeer Zürich / Springer Wien New York. 


\title{
Archipele des Imaginären
}

\author{
mit Beiträgen von \\ Christina von Braun Isolde Charim \\ Peter Fuchs Gerhard Gamm Silvia Henke \\ Jörg Huber Susanne Lüdemann \\ Dieter Mersch Eva Meyer Ivana Müller \\ Michaela Ott K. Ludwig Pfeiffer \\ Alexandra Pontzen Marianne Schuller \\ Gesa Ziemer Simon Zumsteg
}

ith

Institut für Theorie

Theaterhaus Gessnerallee

Siemens Arts Program

Edition Voldemeer Zürich

Springer Wien New York 
Jörg Huber

Institut für Theorie (ith), Departement Kunst \& Medien, Zürcher Hochschule der Künste (ZHdK)

Gesa Ziemer

Institut für Theorie (ith), Departement Kunst \& Medien, Zürcher Hochschule der Künste (ZHdK)

Simon Zumsteg

Deutsches Seminar der Universität Zürich

Institut für Theorie (ith), Departement Kunst \& Medien, Zürcher Hochschule der Künste (ZHdK)

Das Institut für Theorie (ith, Leitung Prof. Dr. Jörg Huber) ist Teil des Departements Kunst \& Medien (Leitung Prof. Giaco Schiesser) der Zürcher Hochschule der Künste (ZHdK, Rektor Prof. Dr. Hans-Peter Schwarz).

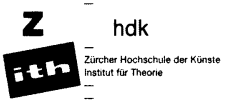

GESSNER

ALLE Siemens artsprogram

Das Werk ist urheberrechtlich geschützt. Die dadurch begründeten Rechte, insbesondere die der Übersetzung, des Nachdruckes, der Entnahme von Abbildungen, der Funksendung, der Wiedergabe auf photomechanischem oder ähnlichem Wege und der Speicherung in Datenverarbeitungsanlagen, bleiben, auch bei nur auszugsweiser Verarbeitung, vorbehalten.

Copyright @ 2009 Institut für Theorie (ith), www.ith-z.ch, und Voldemeer AG, Zürich. Für die Abbildungen Copyright $\odot 2009$ bei den Urhebern bzw. deren Rechtsvertretung.

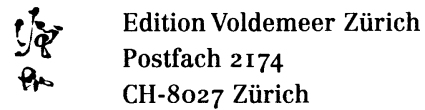

Alle Rechte vorbehalten.

Gestaltung: Edition Voldemeer Zürich

Satz: Marco Morgenthaler, Zürich

Druck: Gebr. Klingenberg Buchkunst, Leipzig

Printed in Germany

SPIN I 2593828

Mit 24 Abbildungen

ISBN 978-3-2 I I-92289-7 Springer-Verlag Wien New York

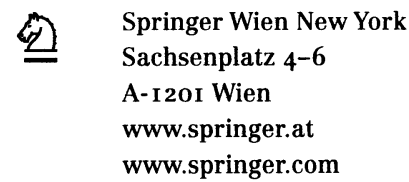




\section{Inhalt}

Simon Zumsteg

Willkommen in den Archipelen des Imaginären

ARCHIPEL DES UNSICHTBAREN

Eva Meyer

Das zusammengefaltete Jetzt

Michaela Ott

Zwang zur Imagination

Susanne Lüdemann

Inversionen des Blicks oder das Unbewusste im Feld des Sehens

Isolde Charim

Der demokratische Glaube

AM THEATERHAUS GESSNERALLEE

\section{Imagine}

Das Imaginäre als Provokation

ARCHIPEL DER REGEL

Dieter Mersch

Positive und negative Regeln:

Zur Ambivalenz regulierter Imaginationen

Marianne Schuller

Déjà-vu oder der nicht-spekuläre Rest

K. Ludwig Pfeiffer

Von der Imagination zum Imaginären (und womöglich zurück) 


\section{ARCHIPEL DES SCHWINDELS}

\section{Gerhard Gamm}

Vom Schwindel: Am Nullpunkt der Erfahrung

Jörg Huber

Devenir cadavre - l'imaginaire: Maurice Blanchot lesen

Silvia Henke

Das Imaginäre ist schwindelerregend:

Ein Versuch im Intermedialen

Christina von Braun

Der Schwindel mit dem Schwindel

\section{ARCHIPEL DER PEINLICHKEIT}

Peter Fuchs

Die Funktion der Peinlichkeit - modern

Gesa Ziemer

Zwischenfälle: Peinlichkeit und Imaginäres auf der Bühne

Alexandra Pontzen

Peinlichkeit und Imagination

Ivana Müller

While We Were Holding It Together (2006) 